\title{
Ciliated Hepatic Foregut Cyst with Obstructive Jaundice
}

\author{
Hidenori Kiyochi ${ }^{a}$ Kenzo Okada ${ }^{a}$ Kazuhide Iwakawa ${ }^{a}$ \\ Mamoru Nakanishi ${ }^{b}$ Hajime Satoh ${ }^{a}$ Shunsuke limoria

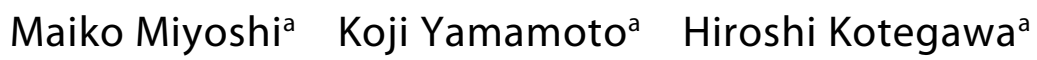

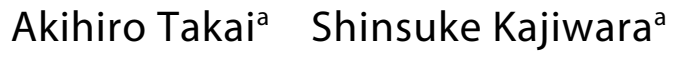 \\ Departments of a Surgery and bPathology, Uwajima City Hospital, Uwajima, Japan
}

\section{Key Words}

Ciliated hepatic foregut cyst - Obstructive jaundice $\cdot$ Porta hepatis

\begin{abstract}
Ciliated hepatic foregut cysts (CHFCs) are rare congenital cystic lesion that are most often solitary, unilocular, and located in the subcapsular region of the medial segment of the left hepatic lobe. The mucoid fluid contents affect imaging studies and often make definitive diagnosis difficult. CHFCs are usually asymptomatic and found incidentally. We report a 69-year-old female patient with a CHFC causing obstructive jaundice, which was difficult to differentiate from a biliary cystic neoplasm. A well-defined cystic lesion measuring $25 \mathrm{~mm}$ in diameter was located in the porta hepatis region. The lesion was densely adherent to the left and right hepatic ducts, riding on the bifurcation, and the common hepatic duct was extrinsically compressed. An extended left hepatectomy was performed. A diagnosis of CHFC was made on the basis of typical histological findings. CHFC should be included in the differential diagnosis for cystic lesions of the liver.
\end{abstract}

\section{Introduction}

Ciliated hepatic foregut cysts (CHFCs) are rare cystic liver lesions that are derived from the remnants of the embryonic foregut [1-3]. In the absence of malignant transformation, these lesions are generally asymptomatic and found incidentally by various abdominal imaging techniques, surgical exploration, or at autopsy [4]. A search of the relevant literature revealed that fewer than 100 cases of $\mathrm{CHFC}$ have been reported since the lesion was first described in 1857 [5]. However, with technological progress and increased usage of various imaging modalities, reports of CHFC have become more frequent during the past two decades. To our knowledge, only two cases of CHFC causing obstructive jaundice have been reported $[6,7]$. We present a case of obstructive jaundice 
caused by compression of the common hepatic duct by a CHFC located at the porta hepatis.

\section{Case Report}

A 69-year-old woman was referred to our hospital with reported abnormal liver function test results and jaundice. Her history was uneventful except for reflux esophagitis. Physical examination revealed mild tenderness in the epigastrium and jaundice of the bulbar conjunctiva. Laboratory studies were significant for abnormal hepatobiliary function: total bilirubin $6.4 \mathrm{mg} / \mathrm{dl}$; direct bilirubin $4.1 \mathrm{mg} / \mathrm{dl}$; aspartate aminotransferase 166 IU/l (normal range 13-33 IU/l); alanine aminotransferase 474 IU/l (normal range 6-27 IU/1); lactate dehydrogenase 261 IU/l (normal range 119-229 IU/l); alkaline phosphatase $223 \mathrm{IU} / \mathrm{l}$ (normal range 115-359 IU/l); gamma-glutamyl transpeptidase 331 IU/l (normal range 6-48 IU/l). CA 19-9 antigen was elevated to $83.8 \mathrm{IU} / \mathrm{l}$ (normal range 0-37 IU/l). The results for other tests, including blood counts, urinalysis, hepatitis $\mathrm{B}$ antigen and hepatitis $\mathrm{C}$ antibody, were normal.

Contrast computed tomography (CT) showed a well-defined, circumferentially enhanced cystic lesion protruding toward the hepatic hilum from the medial segment of the left hepatic lobe. The lesion had no septum and was lower in attenuation than the surrounding liver parenchyma (fig. 1a). Abdominal ultrasonography revealed a hypoechoic cystic mass containing scattered hyperechoic foci and measuring $27 \mathrm{~mm}$ in diameter (fig. 1b). In magnetic resonance imaging (MRI), the lesion exhibited low intensity in T1-weighted images and high intensity in T2-weighted images. The lesion had an isointense component in the dorsal part in both T1- and T2-weighted images (fig. 2a, b). Magnetic resonance cholangiopancreatography showed that the common hepatic duct was extrinsically compressed by the mass at its bifurcation and that the intrahepatic biliary tract was mildly dilated (fig. 2c, d). Hepatic arteriography showed ring enhancement and a feeding artery from the medial branch of the hepatic artery. Endoscopic retrograde cholangiography was unsuccessful.

Since the lesion was causing obstructive jaundice and certain attributes of the cyst suggested malignant transformation (focal cyst wall thickening, circumferential enhancement and the existence of a feeding artery), surgical exploration and excision were undertaken. At this time, serum total bilirubin increased to a peak level of $7.3 \mathrm{mg} / \mathrm{dl}$. However, it decreased to $5.6 \mathrm{mg} / \mathrm{dl}$ just prior to the surgery.

On gross inspection, it was observed that the cystic lesion centered on the porta hepatis region and was densely adherent to the left and right hepatic ducts. Dissection of the adhesion was judged to be impossible. Intraoperative ultrasound imaging indicated that the lesion was riding on the bifurcation of the common hepatic duct and appeared to originate from segment IV of the liver. No marked portal vein invasion was detected. The amount of cystic fluid and the size of the cyst had decreased since the time of admission. An intraoperative cholangiogram performed via the cystic duct visualized the intrahepatic bile ducts relatively well and demonstrated that there was no communication between the cyst and the biliary tree. Under a provisional diagnosis of biliary cystadenoma or adenocarcinoma, an extended left hepatectomy was performed in combination with excision of the suprapancreatic bile duct and node dissection in the hepatic pedicle.

The surgically removed lesion was a $25-\mathrm{mm}$, well-circumscribed, unilocular cyst. The cyst wall was relatively thick, the lining was brownish and smooth, and no solid areas were identified (fig. $3 \mathrm{a})$. Microscopically, the cyst wall was found to consist of the following four layers: inner ciliated pseudostratified columnar epithelium, subepithelial connective tissue, a smooth muscle layer, and an outer fibrous capsule. The surrounding liver parenchyma was normal (fig. 3b). A diagnosis of CHFC was made on the basis of these findings.

The postoperative course was uneventful. The patient's clinical symptoms improved, and the results of hepatobiliary function tests and CA 19-9 antigen returned to normal levels.

\section{Discussion}

The pathogenesis of CHFC is not well understood, but it is considered to be a developmental anomaly of the embryonic foregut. Microscopically, the cyst wall consists of four layers: inner mucin-secreting pseudostratified ciliated columnar epithelium, 
subepithelial connective tissue, a smooth muscle layer, and an outer fibrous capsule. This composition is similar to that of esophageal or bronchogenic cysts derived from the foregut. The cyst is filled with mucoid or viscous fluid secreted by the ciliated epithelium [1-3]. The fluid may contain calcium or blood. The nature and concentration of the fluid contents affect imaging studies and diversify the findings, often making definitive diagnosis difficult.

The differential diagnostic possibilities include simple cyst, parasitic cyst, hepatobiliary cystadenoma, cystadenocarcinoma, and cystic metastatic tumor. In ultrasound images, CHFCs exhibit typical cystic features, including a round shape, a smooth wall, and hypoechoic or anechoic properties with the presence of posterior echo enhancement. An echogenic mass or a mobile hyperechoic region originating in the fluid contents commonly appears in the cyst. A plain CT scan reveals a round lesion with a variable density that reflects the variable fluid viscosities. With T1-weighted MRI, CHFCs show a range of signal intensities, from mildly hypointense to hyperintense, with the signal intensity probably reflecting the fluid viscosity. However, T2-weighted images, in which the intensity is less variable, commonly show the form of a simple cyst with a very high intensity. Caution should be exercised with respect to bleeding of the cyst, which significantly affects intensity and gives rise to hypointense regions in T2-weighted images $[8,9]$.

The shape and the anatomic location of the lesion are also important diagnostic considerations. CHFCs are most often solitary, unilocular, and located in the subcapsular region of the medial segment of the left hepatic lobe, with an average size of $3 \mathrm{~cm}$ [4]. The present case exhibited relatively typical features in imaging studies, which included ultrasound, CT, and MRI. However, it was not typical for benign CHFCs in that the cyst was located in the hepatic hilum and was compressing the common hepatic duct, that it had circumferential enhancement when observed with a CT scan, that there was focal wall thickening on MRI examination, and that the feeding artery was observed by angiography. A diagnosis of biliary cyst adenoma, adenocarcinoma or other cystic malignant tumor could not be excluded and complete excision was needed in our case. A fine needle aspiration biopsy is recommended for a definitive diagnosis of CHFC. The presence of characteristic benign columnar epithelial cells with cilia, identified by an aspiration biopsy, represents definitive diagnostic evidence [10].

CHFCs were originally believed to have no malignant potential, and in some reported cases where a definitive diagnosis was made by aspiration biopsy, there was follow-up but no treatment. However, 3 cases of squamous cell carcinoma arising from CHFC have been reported [11-13]. One patient survived only 2 months after undergoing an operation to remove the carcinoma, indicating that malignant transformation may result in a poor outcome [11]. There is no specific feature associated with carcinomatous transformation, so it is often difficult to distinguish benign CHFC from the malignant form. Serologic tumor markers are of no clinical use as high levels of CA 19-9 have been found in association with benign CHFC, and aspiration cytology may miss areas of malignant transformation. Thus, surgical excision is recommended in most cases of CHFC, particularly when the cyst is larger than $4-5 \mathrm{~cm}$, is symptomatic or expanding, or has a mass formation in the cystic wall [11-13].

According to descriptions of previous cases in the literature, although some patients with CHFC may present with dull upper abdominal pain, most patients have no significant symptoms. However, there have been some prior cases of CHFC causing clinical problems. To our knowledge, excluding cases of malignant transformation, only two cases of obstructive jaundice directly caused by CHFC have been reported in the past. 


\begin{tabular}{r|l|l|l} 
Case Reports $/ \mathrm{n}$ & $\begin{array}{l}\text { Case Rep Gastroenterol 2008;2:479-485 } \\
\text { D01: 10.1159/000176062 }\end{array}$ & Published online: December 2, 2008 & $\begin{array}{l}\text { 2008 S. Karger AG, Basel } \\
\text { ISSN 1662-0631 } \\
\text { www.karger.com/crg }\end{array}$ \\
\hline
\end{tabular}

Dardik et al. reported a $15-\mathrm{cm}$ cyst located on the inferior surface of the right lobe of the liver, distorting the extrahepatic biliary tract [6]. Harty et al. reported an 8-cm predominantly extrahepatic cyst causing portal hypertension and obstructive jaundice through extrinsic compression of the portal vein and common bile duct, respectively [7]. In our patient, the cyst was only $2.5 \mathrm{~cm}$ in diameter. It was, however, located at the porta hepatis, riding on and compressing the bifurcation of the common hepatic duct and thereby causing obstructive jaundice. Other unique features of this case included the decrease in serum total bilirubin level and the slight shrinkage of the cyst in size just prior to surgery. The obstructive jaundice might have been alleviated by reducing the degree of cyst compression of the common hepatic duct. These observations suggest that the cystic fluid volume might have changed.

We have reported a case of CHFC arising in the porta hepatis. The patient presented with obstructive jaundice caused by compression of the common hepatic duct, and the lesion was difficult to differentiate from biliary cystic malignancies. CHFC is usually asymptomatic, but it has the potential for malignant transformation and can be confused radiologically with other entities. Thus, CHFC must be included in the differential diagnosis for cystic lesions of the liver.

Fig. 1. a Contrast CT revealed a well-defined, circumferentially enhanced (arrow) cystic lesion protruding toward the hepatic hilum from segment IV of the liver. The lesion had no septum and was lower in attenuation than the surrounding liver parenchyma. b Abdominal ultrasonography revealed a hypoechoic cystic mass containing scattered hyperechoic foci (arrow) and measuring $27 \mathrm{~mm}$ in diameter.
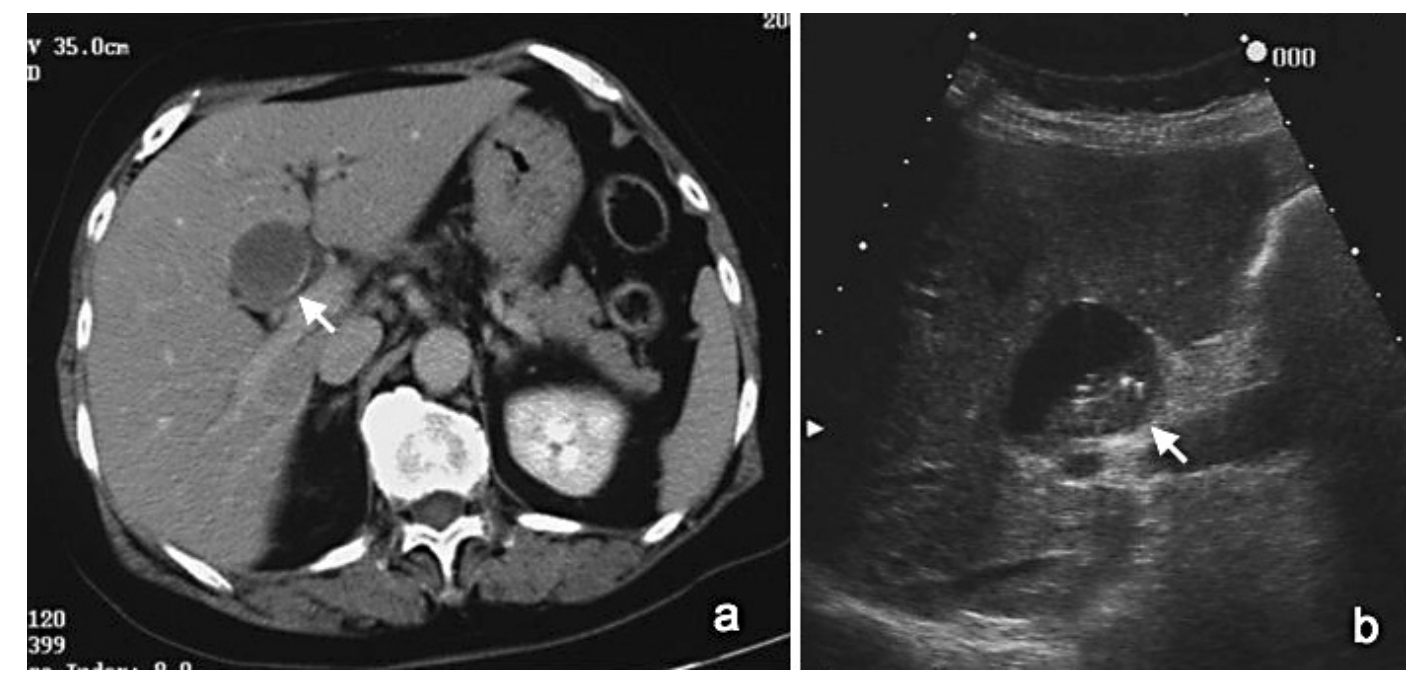


\begin{tabular}{r|l|l|l} 
Case Reports $h$ & $\begin{array}{l}\text { Case Rep Gastroenterol 2008;2:479-485 } \\
\text { Dol: 10.1159/000176062 }\end{array}$ & Published online: December 2, 2008 & $\begin{array}{l}\text { O 2008 S. Karger AG, Basel } \\
\text { ISSN 1662-0631 } \\
\text { www.karger.com/crg }\end{array}$ \\
\hline
\end{tabular}

Fig. 2. In MRI, the lesion exhibited low intensity in T1-weighted spin-echo images (a) and high intensity in T2-weighted images (b). It had an isointensity component in the dorsal part in both T1- and T2-weighted images (arrows) (a, b). Magnetic resonance cholangiopancreatography showed that the common hepatic duct was extrinsically compressed by the mass at its bifurcation and that the intrahepatic biliary tract was mildly dilated $(\mathbf{c}, \mathbf{d})$.

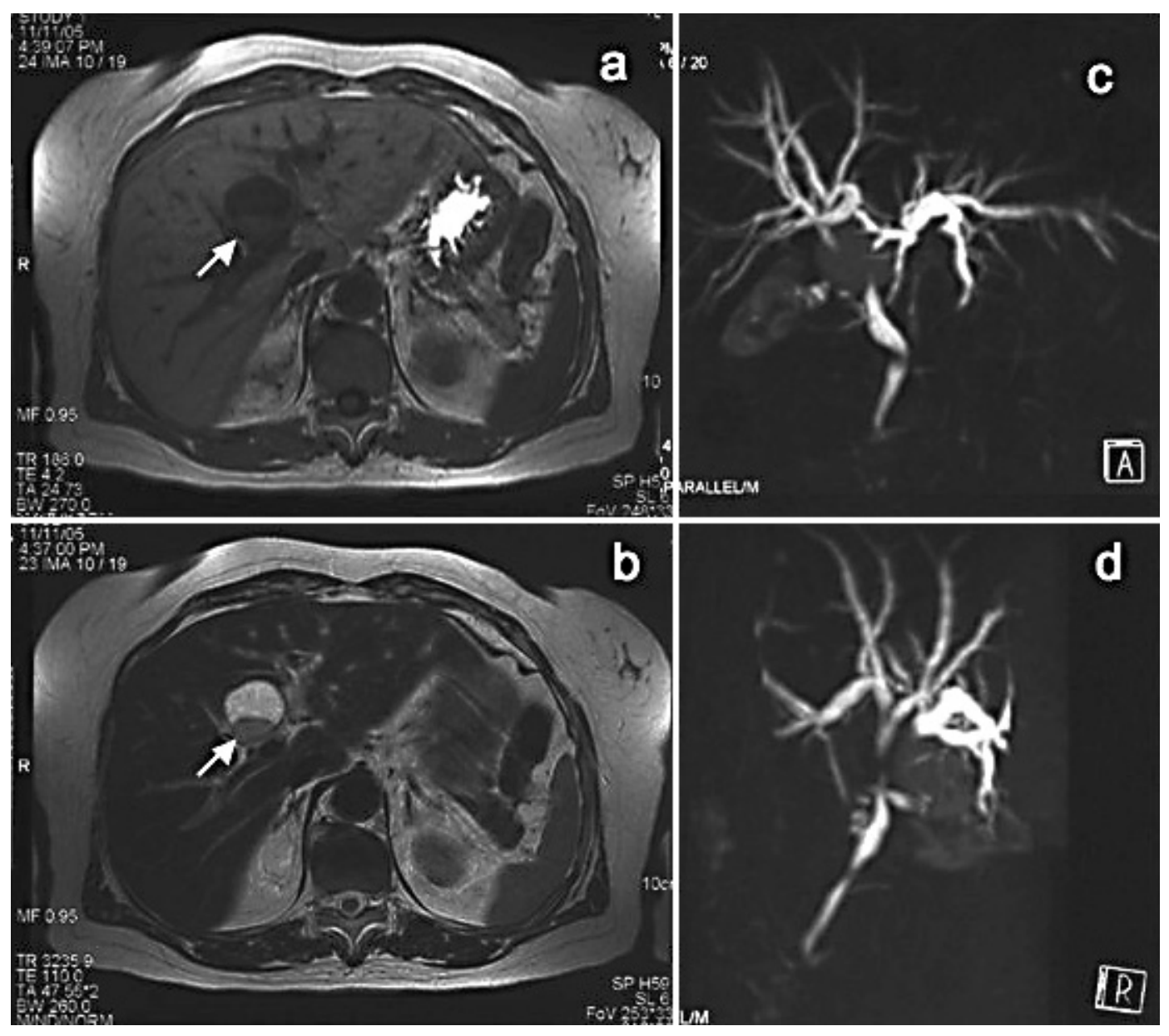




\begin{tabular}{r|l|l|l} 
Case Reports $/ \mathrm{h}$ & $\begin{array}{l}\text { Case Rep Gastroenterol 2008;2:479-485 } \\
\text { D0I: 10.1159/000176062 }\end{array}$ & Published online: December 2, 2008 & $\begin{array}{l}\text { O 2008 S. Karger AG, Basel } \\
\text { ISSN 1662-0631 } \\
\text { www.karger.com/crg }\end{array}$ \\
\hline
\end{tabular}

Fig. 3. a Macroscopic view of the surgical specimen showing a relatively thick cyst wall with a smooth, brownish lining and no solid areas. b Photomicrograph of the surgical specimen showing that the cyst wall comprises inner ciliated pseudostratified columnar epithelium (original magnification, $\times 400$ ).
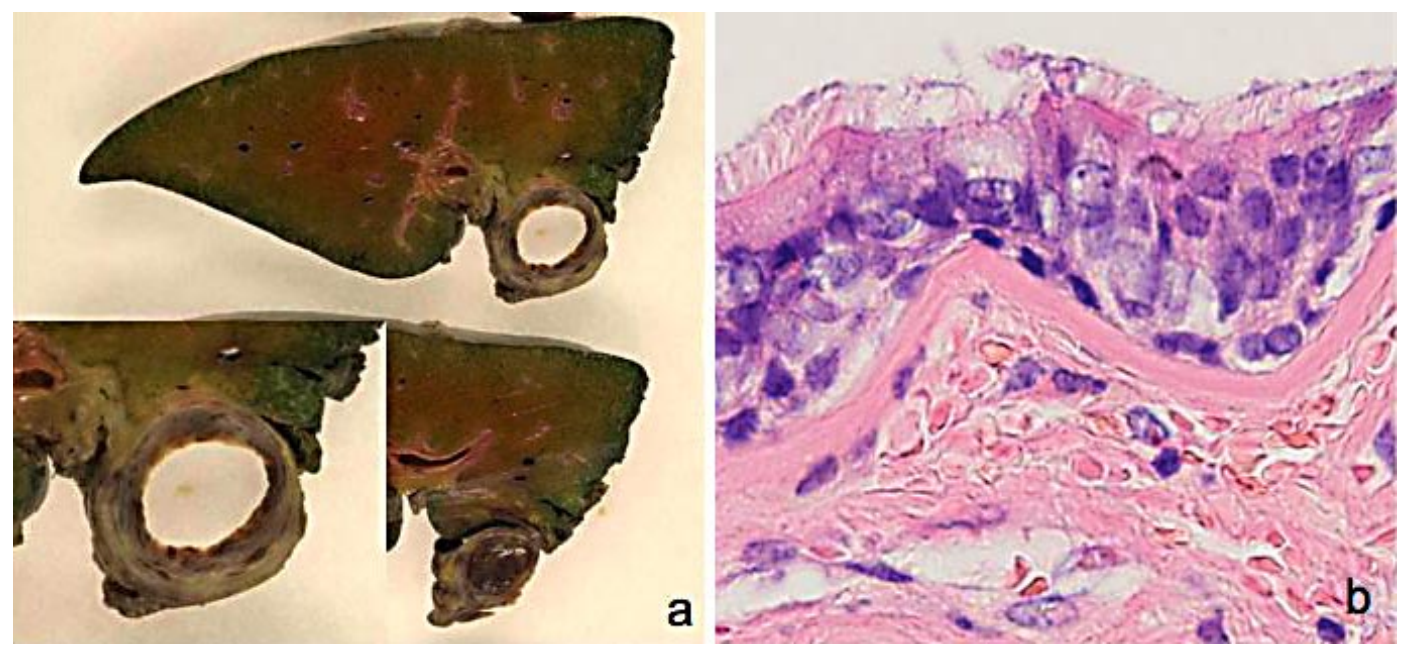


\section{References}

1 Wheeler DA, Edmondson HA: Ciliated hepatic foregut cyst. Am J Surg Pathol 1984;8:467-470.

2 Terada T, Nakamura Y, Kono N, Ueda K, Kadoya M, Matsui O: Ciliated hepatic foregut cyst. A mucus histochemical, immunohistochemical, and ultrastructural study in three cases in comparison with normal bronchi and intrahepatic bile ducts. Am J Surg Pathol 1990;14:356-363.

3 Chatelain D, Chailley-Heu B, Terris B, Molas G, Le Cae A, Vilgrain V, Belghiti J, Degott C, Flejou JF: The ciliated hepatic foregut cyst, an ususal bronchiolar foregut malformation: a histological, histochemical, and immunohistochemical study of 7 cases. Hum Pathol 2000;31:241-246.

4 Vick DJ, Goodman ZD, Deavers MT, Cain J, Ishak KG: Ciliated hepatic foregut cyst: a study of six cases and review of the literature. Am J Surg Pathol 1999;23:671-677.

5 Friedreich N: Cyste mit Flimmerepithel in der Leber. Arch Pathol Anat 1857;11:466-469.

-6 Dardik H, Glotzer P, Silver C: Congenital hepatic cyst causing jaundice: report of a case and analogies with respiratory malformations. Ann Surg 1964;159:585-592.

7 Harty MP, Hebra A, Ruchelli ED, Schnaufer L: Ciliated hepatic foregut cyst causing portal hypertension in an adolescent. AJR Am J Roentgenol 1998;170:688-690.

8 Kadoya M, Matsui O, Nakamura Y, Yoshikawa J, Arai K, Takashima T, Amano M, Kimura M: Ciliated hepatic foregut cyst: radiologic features. Radiology 1990;175:475-477.

-9 Kimura A, Makuuchi M, Takayasu K, Sakamoto M, Hirohashi S: Ciliated hepatic foregut cyst with solid tumor appearance on CT. J Comput Assist Tomogr 1990;14:1016-1018.

10 Hornstein A, Batts KP, Linz LJ, Chang CD, Galvanek EG, Bardawil RG: Fine needle aspiration diagnosis of ciliated hepatic foregut cyst. Acta Cytol 1996;40:576-580.

11 Vick DJ, Goodman ZD, Ishak KG: Squamous cell carcinoma arising in a ciliated hepatic foregut cyst. Arch Pathol Lab Med 1999;123:1115-1117.

12 Furlanetto A, Dei Tos AP: Squamous cell carcinoma arising in a ciliated hepatic foregut cyst. Virchows Arch 2002;441:296-298.

13 Lajarte-Thirouard AS, Rioux-Leclercq N, Boudjema K, Gandon Y, Ramee MP, Turlin B: Squamous cell carcinoma arising in a hepatic foregut cyst. Pathol Res Pract 2002;198:697-700. 